\title{
Art et Politique
}

\section{Reconquérir usage et expérience}

Alice Laguarda

\section{Résumé}

Le développement de pratiques artistiques participatives, relationnelles et contextuelles au sein des sociétés capitalistes démocratiques s'accompagne de multiples modes de relations entre l'artiste et le public. Mais nombre de ces propositions semblent s'être perdues dans la complexité de la matière sociale, propice à la confusion entre animation socio-culturelle, instrumentalisation du public et des artistes ou esthétique compassionnelle. Faut-il en déduire alors que l'artiste est condamné à n'être qu'un professionnel de la communication, qu'un thérapeute des maux de la société ? La poussée des projets et interventions en milieu urbain invite à se demander quelles positions existent aujourd'hui qui permettraient d'évaluer la possibilité d'une fonction critique et polémique de l'artiste. Jochen Gerz nous incite dans Les mots de Paris à réfléchir à la perte de valeur politique de l'espace public. En fondant sa proposition sur la participation d'« exclus » et en ne figeant pas son projet dans une forme conventionnelle, il inverse les règles du jeu artistique et institutionnel, instaure le trouble dans l'appréhension morale de cette «œuvre collective». Nicolas Simarik et Jean Kerbrat élaborent quant à eux des projets qui cherchent à redéfinir l'autorité de l'artiste, invitant le public à construire un usage actif et critique de l'espace public. L'idée d'une résistance apparait, contre les logiques de séparations, par l'élaboration de gestes de "profanation ». Ces propositions nous confrontent au sentiment de perte d'appartenance au monde commun, à l'« envahissement du social » et remettent en question, dans leurs processus comme dans leurs partis pris esthétiques, la relation souvent purement fonctionnaliste qu'entretient aujourd'hui le politique vis-à-vis de l'art. Mots-clés : Art, participation, art relationnel, espace public.

\section{Art et politique}

En ce début de vingt-et-unième siècle, les relations entre art et politique semblent difficiles et distendues. Le poids de l'histoire du siècle passé a engendré une méfiance de l'artiste vis-à-vis du politique, plongeant tour à tour l'art dans l'ère du « micro » (micro-utopies, Nicolas Bourriaud ; micropolitiques, Paul Ardenne) ou du « vide » (vide de sens, vide de relation, vide d'engagement, Gilles 
Lipovetsky). Dans le même temps, certaines propositions - Homeless Vehicles de Krzysztof Wodiczko, série Agonie d'Antoine d'Agata, Madone de Bentalha de Pascal Convert... - semblent avoir dissous les œuvres dans un discours compassionnel, aboutissant à une esthétisation des différents visages de la misère, de la souffrance et de la violence. Ce type d'œuvres ne se borne-t-il pas à énoncer ou représenter de façon littérale l'existence de tel ou tel problème moral ou politique, nous conviant à une déploration sans fin des malheurs du monde?

Les frontières se brouillent entre art et thérapie des maux de la société, art et animation socio-culturelle. Un photographe fait placarder sur des murs ou façades des quartiers difficiles de grandes villes les portraits aux formats monumentaux de ses habitants, symboles d'une communauté humaine dont le sens se trouve miraculeusement restauré. Les festivals des arts de la rue et les biennales d'art dans la ville se multiplient, trahissant souvent une course à l'événementiel et une gestion circonstancielle de la vie de la cité. L'artiste y apparaît comme un multiplicateur de relations, un pourvoyeur de désirs voire un chaman ${ }^{1}$ ! C'est que la demande est forte de favoriser dans certaines propositions institutionnelles, culturelles et politiques la proximité, l'émotion immédiate, les moments de communion populaire, avec le risque de privilégier un seul mode d'expression artistique, une seule catégorie esthétique, un seul usage de l'art. Ne s'agit-il pas en fait de surenchérir sur les forces de socialisation déjà présentes dans nos sociétés sous couvert d'un discours simpliste, issu d'une intention louable, celle qui veut ébaucher une nouvelle «éthique de conviction » à l'âge contemporain, enracinée dans la « reconquête du lien social ${ }^{2}$ »? Si les présences de l'art et de l'artiste dans la cité semblent plus que jamais nécessaires, sous quelles formes doivent-elles apparaitre et s'organiser, quels types de territoires, physiques et symboliques, doivent être investis?

A l'image de Jochen Gerz, Nicolas Simarik et Jean Kerbrat, tous ceux qui tentent de répondre à ces questions se heurtent à leurs dimensions paradoxales.

\footnotetext{
${ }^{1}$ Henri-Pierre Jeudy et Laurence Carré, « L'art social et l'espace public », Programme interministériel de recherche "Culture, ville et dynamiques sociales », février 2000.

${ }^{2}$ Myriam Revault d'Allonnes, L'homme compassionnel, Paris : éd. du Seuil, 2008.
} 


\section{Les mots de Paris : contester les phénomènes de socialisation?}

L'opération Les mots de Paris conçue par Jochen Gerz et parrainée par la Mission $2000^{3}$ est un projet participatif qui a été mené pendant six mois avec une association et un groupe de douze personnes sans travail ni domicile fixe. Ces personnes ont été salariées par l'association «Aux Captifs, la Libération » fondée par un prêtre du diocèse de Paris et qui travaille avec des lieux d'accueil de jour. Plusieurs étapes ont préparé le projet jusqu'à l'installation finale prenant la forme d'un abribus de la RATP posé sur le parvis de l'Église Notre-Dame-deParis et d'une grande boîte de verre au sol où l'on pouvait lire un texte écrit en commun avec les participants. Sur les vitres de l'abribus était inscrit le même texte en neuf langues : «Si vous n'avez rien à donner, parlez. Si vous n'avez rien à dire, donnez ». Les passants étaient invités à déposer de l'argent dans une fente visible sur la plaque de verre pour aider à l'élaboration d'un programme de réinsertion sociale. Près de quinze mille euros ont été récoltés et ont servi à l'association et à l'accompagnement des membres de l'équipe. Les mots de Paris a provoqué de nombreuses polémiques dans la presse sur l'utilisation à des fins artistiques de personnes socialement précaires, voire désocialisées. Dans le journal L'Humanité du 27 juin 2000, Jacques Moran écrivait :

«L'exposition humaine est depuis longtemps une réalité. À la fin du siècle dernier, les pays coloniaux exhibaient des Africains à l'Exposition universelle de Paris (...). L'exhibition humaine à des fins artistiques n'est-elle que la sublimation de pratiques ancestrales (l'esclavage), sans intérêt autre que la satisfaction narcissique d'un auteur occidental nanti ? À quoi les artistes rétorquent que (...) l'utilisation de modèles vivants est la condition sine qua non d'une sur-représentation de ce que la société dissimule du mieux qu'elle le peut, en isolant ses victimes de ceux qu'elle n'a pas encore rejetés ».

De nombreux commentateurs insistèrent quant à eux sur la dimension démagogique du projet, fustigeant son engagement « frivole et circonstanciel», son côté « petites sœurs de la Charité et bonne conscience à deux sous (ou plutôt, tout compris, selon budget prévisionnel établi par l'artiste, à 1,46 MF), sa nullité

\footnotetext{
${ }^{3}$ Mission mise en place par le ministère de la Culture et de la communication pour la célébration de l'an 2000 qui a financé des dizaines de projets artistiques et culturels partout en France (exposition La Beauté en Avignon, célébration de l'architecture contemporaine à Bordeaux, ouverture du musée international des arts modestes dans la ville de Sète, concerts, etc.).
} 
esthétique », son côté « relationnel chic et $\operatorname{choc}^{4} »$. Au-delà des anathèmes, ce projet de Jochen Gerz nous semble surtout relever d'une réflexion politique et sans nul doute polémique -, celle de la distinction entre domaine public et domaine privé posée par les Grecs anciens qui accordaient à l'homme deux ordres d'existence : ce qui lui est propre, ce qui le particularise ; ce qui est commun à tous. Le privé, domaine de la nécessité, de la subsistance individuelle et de la perpétuation de l'espèce, renvoie aux activités d'entretien de la vie biologique. Sa localité est l'espace du foyer (oikos), de la famille. Le domaine public se veut le domaine de la liberté, de la parole, de l'échange, l'espace de la cité. C'est l'espace politiquement commun à tous les hommes, dans lequel on doit accomplir de grandes choses aux yeux de tous, susciter l'admiration publique. L'homme y réalise son humanité parce qu'il le fait devant les autres et surtout parce que ce qu'il fait est dégagé de la soumission aux nécessités de la vie biologique: "C'est la présence des autres voyant ce que nous voyons, entendant ce que nous entendons, qui nous assure de la réalité du monde et de nous-mêmes ${ }^{5}$. »Selon Jochen Gerz,

«ce qui est caché doit être vu. Le travail de l'artiste est de montrer ce qui est, ne pas faire du beau, mais un acte de présence. Je ne chante pas le pauvre, je le montre, sans distance poétique ${ }^{6}$.»

L'artiste a donc choisi de se référer à la figure mythique du mendiant qui joua longtemps le rôle pastoral de conteur et de fou, celui de l'« exclu» qui vit hors du monde et révèle, depuis sa marginalité, ce qu'est la communauté. Les mots de Paris invite ainsi à voir ce que l'on ne peut pas voir, à opérer un retournement de situation. Les actions demandées aux participants (faire la manche, rester sous l'abribus, faire part de leurs difficultés sociales, de leur sentiment d'exclusion, aller vers le public, se mettre en scène), jouent constamment sur le brouillage entre ce qui relève, précisément, du privé et du public et aussi du religieux et du non religieux. Les inversions et décalages établis par Jochen Gerz engendrent une crise de notre vision idéalisée de l'espace public : car comme le fait remarquer Judith Butler,

\footnotetext{
${ }^{4}$ Paul Ardenne et Élisabeth Wetterwald, «Jochen Gerz, portrait de l'artiste en démagogue », in journal parpaings $\mathrm{n}^{\circ} 14$, juin 2000 , éd. Jean-Michel Place.

${ }_{5}^{5}$ Hannah Arendt, Condition de l'homme moderne, 1958, 1961 pour la traduction française, Paris : éd. Calmann-Lévy.

${ }^{6}$ Jochen Gerz, «L'art, violence de la mémoire », entretien in revue art press $\mathrm{n}^{\circ} 179$, avril 1993.
} 
«le public peut-il jamais être constitué comme tel sans une certaine population reléguée dans le privé et donc dans le prépolitique, et ceci n'est-il pas radicalement inacceptable pour toute vision démocratique radicale ${ }^{7}$ ?»

Pour qu'il y ait un espace public fort, faut-il nécessairement que certains en soient exclus, demeurent à l'écart ? Ceux qui ont été " relégués », qui sont désignés et considérés comme les « exclus » du système économique, social et politique, sont devenus les acteurs du projet de Jochen Gerz, partis, donc, à la conquête de l'espace public. Mais dans le même temps, ils se retrouvent dans un espace totalement mis en scène, on leur fait jouer une comédie, on plonge dans le simulacre. Si le projet de l'artiste fait apparaitre le refoulé social incarné par les « exclus », les marginaux, il entend aussi leur faire symboliser une communauté originelle des hommes qu'il s'agit de recréer, voire de sacraliser, de solenniser, au cœur du territoire urbain. Cette tentation du sacré, écho à la thèse d'un « tournant pastoral » de l'art contemporain ${ }^{8}$, est analysée par Henri-Pierre Jeudy et Laurence Carré. Les auteurs remarquent que certaines pratiques artistiques contemporaines mettent en avant une gloire du quelconque, où « l'ordinaire devient beau comme trace du vrai ; et il devient trace du vrai si on l'arrache à son évidence pour en faire un hiéroglyphe, une figure mythologique ou fantasmagorique $^{9}$.» C'est semble-t-il ce qu'a cherché à faire Jochen Gerz. Mais l'esthétisation inévitable qui s'ensuit peut également être synonyme de dépolitisation. On attend ainsi des artistes qu'ils deviennent des «super agents de développement local », des « professionnels de la communication », des «multiplicateurs de relations ». L'artiste ne risque-t-il pas, alors, de se transformer en thérapeute des maux de la ville, des crises sociales et économiques, venant combler miraculeusement les faillites du politique?

\section{L'urbanité en déroute}

En résidence dans le quartier Empalot de Toulouse, Nicolas Simarik a été invité en 2005 par l'association «Entrez sans frapper» qui organise des résidences d'artistes visant à impliquer les habitants d'un quartier dans la production d'une

${ }^{7}$ Judith Butler, conversation avec Gayatri Chakravorty Spivak, L'État global, Paris : Payot, 2007.

${ }^{8}$ Tristan Trémeau et Amar Lakel identifient un «tournant pastoral » de l'art contemporain dans leur intervention lors du colloque international L'art contemporain et son exposition, Centre Georges Pompidou, Paris, octobre 2002.

${ }^{9}$ Henri-Pierre Jeudy et Laurence Carré, « L'art social et l'espace public », art. cit. 
œuvre collective. Le projet consiste à détourner le catalogue de La Redoute, symbole en France de la vente par correspondance. L'objet final est un catalogue de 1236 pages tiré à six mille exemplaires, reprenant la mise en page et la hiérarchie d'information du catalogue originel. Intitulé La Déroute, le projet a l'ambition de mettre en valeur, par l'image, la vie quotidienne d'un quartier.

Le catalogue est conçu comme un travail artistique collectif qui place au cœur du projet les liens d'une population sur un territoire urbain secoué de crises successives. À la place des prix des produits indiqués dans le catalogue originel, on peut lire l'heure des séances photographiques et les descriptifs qui ne concernent plus les produits habituels (vêtements, mobilier, vélos, motos, jouets...) mais les noms des six cents mannequins volontaires. Les slogans et fausses marques de produits : "Pose toujours», "Melrose et Place », "Tittsoeur», «Côté humain », « Extensible, mon quartier grandit avec moi! !, « Avancer sans rivalité », «Zest de la déroute », traduisent la volonté des habitants de s'approprier et de détourner le vocabulaire de la publicité. Il s'agit de redonner la possibilité d'un usage commun de l'espace public : un usage critique et non pas passif, infantilisant, strictement consommatoire.

Comme le remarque l'artiste, le besoin de réel existe toujours, dans une société de consommation de masse aux stratégies commerciales et publicitaires très agressives. Le détournement de l'objet catalogue met ainsi en scène une exagération du bonheur consommatoire, révélateur de comportements sociaux et culturels universels. Il y a dans la proposition de Nicolas Simarik une interrogation sur la notion d'usage, assez proche nous semble-t-il de la réflexion qu'élabore le philosophe italien Giorgio Agamben dans son ouvrage Profanations. Le développement du système capitaliste au vingtième siècle, la consommation telle qu'elle se manifeste dans sa phase extrême (domination de la logique utilitariste) ne semble plus autoriser aucune critique ni aucun usage. L'homme a perdu la possibilité de s'inscrire au sein d'un monde commun. L'enjeu pour les hommes, et pour les artistes serait alors de reconquérir ou de changer l'usage (d'un territoire, du monde, des symboles, du sacré, de l'oeuvre d'art...) afin d'échapper à la violence, à la négation de l'humanité de l'homme. Or, pour Agamben, la société capitaliste nous place dans une religion du désespoir. C'est le "procès de séparation» :

«Et tout comme dans la marchandise, la séparation fait partie de la forme même de l'objet qui se scinde en valeur d'usage et en valeur d'échange pour se transformer en un fétiche insaisissable, de la même manière, tout ce qui désormais se trouve fait, produit et vécu 
(le corps humain lui-même et la sexualité et le langage aussi) est comme séparé de soi et disloqué dans une sphère distincte qui ne définit plus aucune division substantielle et où tout usage devient durablement impossible ${ }^{10} »$.

Cette sphère, c'est la consommation :

«Si les consommateurs sont malheureux dans les sociétés de masse, ce n'est pas seulement parce qu'ils consomment des objets qui ont incorporé leur inaptitude à l'usage, mais aussi et surtout parce qu'ils croient exercer sur eux leur droit de propriété, parce qu'ils sont devenus incapables de les profaner ${ }^{11}$.»

Le philosophe s'approprie de façon audacieuse la notion de profanation. Profaner consiste à désacraliser un usage ancien, en le rendant inefficace, à abolir et effacer les séparations sociales, politiques, économiques, à apprendre à en faire un nouvel usage, à jouer avec elles. Avec le détournement du catalogue de La Redoute proposé par Nicolas Simarik, il s'agit bien de créer un nouvel usage d'un objet, d'un produit qui s'inscrit totalement dans la logique marchande capitaliste et uniformise les désirs et les comportements. L'artiste pointe les logiques de séparations qui traversent nos sociétés et sont particulièrement visibles et éprouvées sur les territoires urbains. L'action permanente de ces logiques fait vivre à l'homme « l'expérience angoissante de la destruction de tout usage possible. » Angoissante parce que si l'homme est dépossédé de tout usage possible, il n'y a plus d'espace commun possible, plus de monde humain commun possible. La notion d'usage doit être ici bien distinguée de deux dérives. Ce n'est pas l'assimilation de l'usage à la consommation des choses (frénésie qui domine la société actuelle) : «La consommation qui détruit nécessairement la chose n'est rien d'autre que l'impossibilité ou la négation de l'usage qui présuppose que la substance de la chose reste intacte ${ }^{12} »$. Ceci veut dire que dans l'usage dont il est question, les choses ne sont pas usées, utilisées, mais au contraire toujours présentes. Il ne s'agit pas non plus d'assimiler l'usage à la propriété : «L'usage est toujours une relation avec ce qu'on ne saurait s'approprier, il se réfère aux choses en ce qu'elles ne peuvent pas devenir un objet de possession ${ }^{13}$ ». L'usage ne consiste pas à s'approprier mais à partager. Le nouvel usage dont il est ici question, suivant la réflexion de Giorgio Agamben, correspond à « une

\footnotetext{
${ }^{10}$ «Éloge de la profanation », in Profanations, Paris : éd. Rivages, 2005.

${ }^{11}$ Idem.

12 "Éloge de la profanation », in Profanations, Paris : éd. Rivages, 2005.

${ }^{13}$ Idem.
} 
praxis qui s'est libérée de sa relation à une fin, a oublié joyeusement son objectif et peut désormais s'exhiber comme telle, comme un moyen sans fin ». Le paradoxe de cet usage, c'est qu'il n'use pas. Il laisse le monde intact. Qu'est-ce qui reste intact? Quelle attitude permet de l'atteindre?

\section{Retrouver l'usage, retrouver le politique}

Jean Kerbrat a réalisé plusieurs projets participatifs avec des habitants de la ville de Calais - invité par le Channel, Scène nationale -, restant de longs mois en résidence dans la ville. Dans Calais Kerbrat. On gagne au change (1999), une annonce rédigée par l'artiste est placardée dans la ville, diffusée à la radio et dans la presse locale :

«Prêtez-moi un de vos objets pendant un mois, je le transformerai gratuitement. Il sera exposé parmi tous les autres dans une galerie d'art dans le centre-ville de Calais, où vous pourrez ensuite le récupérer signé avec sa plus-value. »

Les objets prêtés à l'artiste - une centaine : assiettes, balles de tennis, piano, voiture, jouets, bibelots, bandonéon, figurines, porte-monnaie... - font l'objet de diverses transformations : greffes, excroissances, embellissements, perturbations. Ils sont ensuite présentés dans la galerie et restitués aux propriétaires qui le souhaitent. Le projet intègre la dimension affective propre à l'histoire de chaque objet : ils représentent en effet autant de fragments d'histoire, de traces du réel social et urbain. Comme le fait remarquer l'historien d'art Paul Ardenne, chaque objet est une matière de vie qui se trouve revivifiée par le geste de création, reproblématisée par le geste d'esthétisation.

Une réflexion sur l'usage se construit : les objets restitués font apparaître la «plus-value ${ }^{14}$ acquise par ces derniers. Une plus-value d'un nouveau genre, puisqu'elle privilégie l'idée d'un échange équitable entre l'artiste et le propriétaire de l'objet transformé. L'échange n'est plus basé sur le principe marchand et le profit, il reconduit une forme de troc, instituant un nouveau mode de relation entre l'artiste et les Calaisiens. C'est que Jean Kerbrat veut aussi interroger les formes très en vogue de l'art participatif ou relationnel. Dans On gagne au change, le public est invité à se confronter à l'art, à en accompagner les étapes, à vivre une expérience symbolique et non pas à subir une forme autoritariste, condescendante, du regard de l'artiste sur le réel et les autres, les «non-initiés ». En

\footnotetext{
${ }^{14}$ Paul Ardenne, Calais Kerbrat. Tyranniser la plus-value, texte du catalogue de l'exposition, Le Channel, Scène nationale de Calais, 2000.
} 
2001, c'est l'opération La Coupe déborde. Partant de l'idée de donner à Calais sa coupe de France, Jean Kerbrat propose d'organiser et de mettre en scène des matches de baby-foot dans l'espace de la galerie du Channel, du tirage au sort des équipes jusqu'à la remise de coupes spécialement fabriquées par l'artiste. Il installe dans la galerie un espace de gradins avec des chaises surélevées, un baby-foot, une buvette. Autour de la scène des gradins, des vidéos sont diffusées qui condensent des extraits d'images de matches et de réactions de supporters. Une seconde salle accueille une vidéo, montage d'images d'archives, extraits d'émissions de télévision, de spots publicitaires interprétés par des joueurs de foot, images de guerre, jingles publicitaires... Dans cette même salle, au sol, sont présentés des ballons de football en papier journal fabriqués par des enfants de Calais avec l'artiste. Les matches se déroulent pendant trois mois. Les membres des clubs de supporters existant à Calais se sont inscrits, ainsi que des champions de baby-foot et des équipes féminines. Toutes les règles sont perturbées. La salle principale, avec ses chaises-gradins, évoque l'espace d'un tribunal. Les hurlements agressifs qui proviennent des vidéos diffusées autour des gradins créent une surenchère sur un climat d'excitation déjà présent à chaque match. Le baby-foot a remplacé le football. Dimension de célébration festive et évocation dans les vidéos des dérives du milieu du sport (le «Mercato », la violence des supporters) se mêlent. Sommes-nous bien dans un jeu ? Par ce détournement des règles et ce travail de mise en scène, Jean Kerbrat nous renvoie à l'une des réalités de nos sociétés, celle des ersatz : le football, ersatz de religion, fantasme d'une communauté humaine soudée, solidaire, en fait divisée en «tribus » très hostiles les unes aux autres. Ersatz encore, à un autre degré, dans les coupes créées par l'artiste. Les trophées sont ici totalement métamorphosés, allongés, augmentés de greffes, envahis de moules frites, désacralisés. Un jeu complexe nous est proposé avec les objets qui nous entourent et la relation qu'on peut créer avec eux. Dans La coupe déborde, Kerbrat exacerbe la métaphore sportive pour en dénoncer les dérives : culte de la performance, fantasme de l'équipe, deux termes devenus des valeurs en soi, largement récupérés dans les discours politiques et le monde de l'entreprise, sans grande sincérité. Jouant dans l'exposition de la galerie du Channel sur les effets d'abondance, de tropplein, de surenchère, puis de vides, de décalages, Kerbrat interroge l'impact des figures sportives dans la société et les «affaires » qui y sont reliées. Ces phénomènes et ces pratiques sont pour l'artiste les révélateurs d'une décomposition de la communauté politique, de la communauté humaine, l'expression des forces de socialisation. Proposition de l'artiste, en retour : être celui qui transforme, déplace les règles qui tendent à normaliser, à faire marcher droit ou à décomposer la communauté. Transformer l'usage de certains clichés, certains symboles, en les manipulant esthétiquement et idéologiquement. Ce geste qui désacralise les codes du monde du football et la puissance de ses intérêts économiques in- 
duit aussi une réflexion sur l'autorité de l'artiste. La relation que cherche à instaurer Jean Kerbrat avec le réel et le public nous semble créer de la distance critique, parce qu'elle repose sur ce travail de manipulation esthétique. Selon Jean Kerbrat,

«Il y a une nécessité absolue de créer de la fiction, relative à la nécessité d'introduire une distance, de rompre avec une certaine proximité. (...) Pour qu'il y ait une efficacité politique, pour que cela soit un acte de résistance, il faut regarder Le Réel et non pas la réalité : c'est déjà un réel transformé, une autre image du réel ${ }^{15}$. »

C'est là la condition à laquelle il peut y avoir «transformation esthétique du réel », et non-esthétisation du réel. Ceci est très important. Car nous vivons, selon la formule de Christian Ruby, dans un «État esthétique » qui cherche à redonner au lien social une puissance symbolique fondée sur un fantasme d'unité et de sacré.

\section{Conclusion}

Ainsi, l'art contemporain peine à reconquérir usage et expérience, écartelé entre sa défiance vis-à-vis de tout discours qui introduirait dans les œuvres un contenu politique trop monolithique ou une visée compassionnelle, et l'éclatement des pratiques en singularités isolées les unes des autres. Comment, alors, envisager la relation entre art et politique ? L'une des pistes réside sans doute dans la continuité de ce retour à l'usage tel que le revendiquent Nicolas Simarik ou Jean Kerbrat et dans la conception d'un art refusant toute instrumentalisation :

« Pour nous, la politique, en effet, n'a pas pour vocation de substituer formellement des œuvres au désoeuvrement social et politique, de substituer la citation d'œuvres aux grands récits désagrégés de l'histoire ou de dispenser l'assurance naïve qu'une communication étendue peut raffermir le "lien social". Soumise à ces pratiques, elle ne correspond à rien d'autre qu'à une technique de gestion de bonheurs consommatoires. Ce que nous appelons la politique définit plus exactement le moment, toujours renouvelé et extensible, où les citoyens ont droit à des expériences et des projets, à une histoire qui est encore à accomplir ${ }^{16}$. »

\footnotetext{
${ }^{15}$ Propos de l'artiste sur son site internet : www.kerbratjean.fr

${ }^{16}$ Christian Ruby, L'État esthétique. Essai sur l'instrumentalisation de la culture et des arts, Bruxelles : éd. Labor, 1999. Aline Caillet, « La convivialité, une esthétique relation-
} 
Dans nos sociétés, on choisit bien souvent de ne voir dans la quête de proximité et de convivialité qu'une "affection privée », une relation forcément positive et favorable qui évince toute possibilité de conflits et de débats dans la sphère publique. On oublie la médiation nécessaire d'un idéal commun qui doit être débattu, «comme s'il suffisait de résoudre les difficultés d'une politique dans une pharmacopée » sociale et esthétique, «administrée depuis ses lieux de manifestation ${ }^{17}$.»

Les exemples d'interventions artistiques dans la cité que nous avons examinées sont au cœur de ces problématiques. Pour une orientation qui leur est commune et qui consiste, au-delà des illusions et ambiguités (nombreuses, on l'a vu), à mettre ce que nous appelons « réalité » à distance. Découvrir, faire advenir le réel sous la réalité apparente, c'est bien explorer notre humanisation du réel (l'expression d'une subjectivation du réel) qui nous est spécifique et qui est, elle aussi, double, changeante, jamais figée. Explorer cette existence humaine du réel correspond à une vocation salutaire de l'art contemporain, proprement politique : "Vivre ensemble dans le monde, c'est dire essentiellement qu'un monde d'objets se tient entre ceux qui l'ont en commun, comme une table est située entre ceux qui s'assoient autour d'elle; le monde, comme tout entredeux, relie et sépare en même temps les hommes ${ }^{18}$.»

\section{Remerciements à Jean Kerbrat et Didier Malgor.}

nelle ?», in journal parpaings ${ }^{\circ} 23$, mai 2001, éd. Jean-Michel Place. Hannah Arendt, Condition de l'homme moderne, op. cit.

${ }^{17}$ Aline Caillet, «La convivialité, une esthétique relationnelle ?», in journal parpaings $\mathrm{n}^{\circ} 23$, mai 2001, éd. Jean-Michel Place.

${ }^{18}$ Hannah Arendt, Condition de l'homme moderne, op. cit. 